\title{
Over-Complete Source-Mapping Aided AMR-WB Using Iteratively Detected Differential Space-Time Spreading
}

\author{
N. S. Othman, M. El-Hajjar, A. Q. Pham, O. Alamri, S. X. Ng and L. Hanzo* \\ School of ECS, University of Southampton, SO17 1BJ, UK. \\ Tel: +44-23-8059 3125, Fax: +44-23-8059 4508 \\ Email: lh@ecs.soton.ac.uk, http://www-mobile.ecs.soton.ac.uk
}

\begin{abstract}
The achievable performance of a jointly optimised iterative source and channel decoding (ISCD) arrangement invoking the Adaptive MultiRate Wideband (AMR-WB) speech codec is characterized, which exploits the intentional redundancy imposed by the proposed Over-Complete source-Mapping (OCM) scheme. The resultant OCM-aided AMR-WB bitstream is protected by a Recursive Systematic Convolutional (RSC) code and mapped to a Differential Space-Time Spreading (DSTS) arrangement using Sphere Packing (SP) modulation for transmission over narrowband temporally correlated Rayleigh fading channels. The effect of appropriately apportioning the total amount of redundancy between the source and channel codecs on the attainable system performance is demonstrated, while keeping the overall coding rate constant. The decoding convergence of the proposed scheme is studied with the aid of Extrinsic Information Transfer (EXIT) charts. Explicitly, our experimental results show that the specific scheme using a 2/3-rate channel encoder and a 3/4-rate OCM scheme exhibits an $E_{b} / N_{0}$ gain of $0.7 \mathrm{~dB}$ at the SegSNR degradation point of $1 \mathrm{~dB}$, when compared to the system that assigns all the redundancy to the OCM scheme. By contrast, the scheme using a 3/4-rate channel encoder and a 8/9-rate OCM results in an $E_{b} / N_{0}$ gain of $\mathbf{1 . 0} \mathbf{d B}$.
\end{abstract}

\section{MOTIVATION AND BACKGROUND}

A realistic, finite-delay lossy source codec leaves some residual redundancy in the encoded parameters, which is not the case for Shannon's ideal entropy codec. Fortunately, this residual redundancy may be beneficially exploited by joint source-channel coding for the sake of providing error protection [1].

It was demonstrated in $[2,3]$ that the innovative concept of soft speech bits employed in the Iterative Source and Channel Decoding (ISCD) scheme of [4] can be further improved by exploiting both the intentionally imposed and the inherent unintentional residual redundancy found in source encoded bitstream. The performance of the ISCD scheme of [2] was characterised using ten Brink's EXIT charts [5].

Recently, it was demonstrated in [6] that the performance of the proposed scheme can be further improved by employing an enhanced Over-Complete source-Mapping (OCM) aided soft-bit assisted AMRWB decoder. By contrast, in this contribution we propose and investigate the jointly optimised ISCD scheme of Figure 1 by partitioning the total available bit-rate budget between the source and channel codecs. More explicitly, we propose a jointly optimised ISCD arrangement invoking the Adaptive MultiRate-Wideband (AMR-WB)

The financial support of the Universiti Tenaga Nasional Malaysia, of Vodafone under the auspices of the Dorothy Hodgkin Postgraduate Award and that of the EPSRC, UK as well as that of the European Community under Seventh Framework Programme grant agreement ICT OPTIMIX nINFSO-ICT214625 is gratefully acknowledged. speech codec [7] exploiting the intentionally increased residual redundancy of the AMR-WB encoded bitstream by using the novel OCM of [8]. The resultant OCM aided bit-stream is protected by a Recursive Systematic Convolutional (RSC) code and transmitted using Differential Space-Time Spreading (DSTS) aided Sphere Packing (SP) modulation [9] for attaining a diversity gain without the need for any high-complexity MIMO channel estimation. This three-stage system is termed as the DSTS-SP-RSC-AMRWB-OCM arrangement.

The outcome of the rest of this paper is as follows. In Section 2, the overall system model is described, while our EXIT chart analyis is provided in Section 3. Section 4 characterizes the achievable performance of our proposed three-stage scheme, leading to our conclusions in Section 5.

\section{SYSTEM OVERVIEW}

The DSTS-SP-RSC-AMRWB-OCM system model is depicted in Figure 1. At the transmitter side, the speech signal is encoded at $23.05 \mathrm{kbps}$ using the AMR-WB speech codec. The AMR-WB speech codec is capable of supporting nine different bit rates [7]. Each AMR-WB frame represents $20 \mathrm{~ms}$ of speech, producing 461 bits at the bit rate of 23.05 kbps.

In the advocated system we employ the over-complete sourcemapping philosophy of [8]. More explicitly, the AMR-WB encoded bit-stream is divided into $M$ bits/symbol sequence using the OCM of rate $M / N$, where $N$ denotes the number of bits corresponding to an OCM symbol. For example, when using the OCM of rate $3 / 4$, the AMR-WB encoded bit-stream is divided into 3-bit source symbols $\tilde{\mathbf{v}}_{\kappa, \tau}=\left[\tilde{v}(1)_{\kappa, \tau} \tilde{v}(2)_{\kappa, \tau} \ldots \tilde{v}(M)_{\kappa, \tau}\right]$, where $M=3$ is the total number of bits assigned to the $\kappa$ th parameter, with $\kappa$ denoting the index of each 3-bit source symbols. Then, $\tilde{\mathbf{v}}_{\kappa, \tau}$ is mapped to the bit sequence, $\mathbf{u}_{\kappa, \tau}=\left[u(1)_{\kappa, \tau} u(2)_{\kappa, \tau} \ldots u(N)_{\kappa, \tau}\right]$ using over-complete source-mapping, where $N=4$. Subsequently, the outer interleaver, $\pi_{\text {out }}$ permutes the bits of the sequence $\mathbf{u}$, yielding $\tilde{\mathbf{u}}$ of Figure 1, which are then protected by RSC encoder. Next, the RSC encoded bits are interleaved by interleaver $\pi_{i n}$ of Figure 1. Subsequently, the SP mapper maps $B$ number of channel-coded bits $\tilde{\mathbf{c}}=\left[\begin{array}{llll}\tilde{c}_{0} & \tilde{c}_{1} \ldots & \tilde{c}_{B-1}\end{array}\right]$ $\in\{0,1\}$ to a SP symbol $x \in X$ as detailed in [9]. Then the SP modulated symbols are transmitted using DSTS via two transmit antennas.

The complex-valued received symbols $\mathbf{z}$ are demapped to their Logarithmic-Likelihood Ratios (LLR) representation for each of the $B$ number of RSC-encoded bits per DSTS-SP symbol. Then, iterative demapping/decoding is carried out between the SP demapper, the RSC decoder and the Soft-Bit Source Decoding (SBSD) [4] decoder. The iterative process is performed for a number of consecutive iterations. The inner iterative loop corresponds to the iterations between the SP demapper and the RSC decoder, while the outer iterative loop represents the extrinsic information exchange between the SBSD decoder and the RSC decoder. The variable $L($.$) in Figure 1$ represents the LLRs of the bits. The notations $\tilde{c}, c, \tilde{u}$ and $u$ in the round brackets (.) of Figure 1 denote the SP bits, RSC coded bits, RSC data bits and 

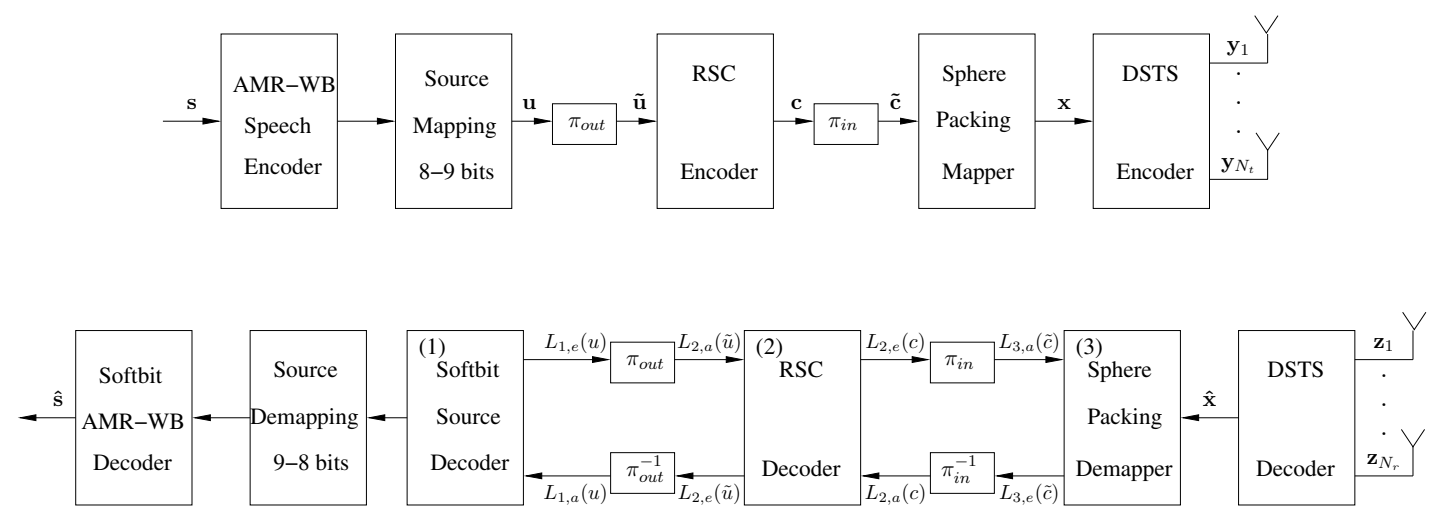

Figure 1: Block diagram of the DSTS-SP-RSC-AMRWB-OCM scheme.

the OCM aided AMR-WB encoded bits, respectively. The LLRs $L_{., a}$, $L_{., p}$ and $L_{., e}$, denote the a priori, a posteriori and extrinsic information of Figure 1. The LLRs of the three decoders are differentiated by the corresponding subscripts (.) of $\{1,2,3\}$ in the decoder of Figure 1.

We introduce the term "system iteration" defined as two inner iterations between the SP demapper and the RSC decoder followed by one outer iteration between the RSC and the SBSD decoders. In the proposed scheme, the SBSD decoder exploits the natural residual redundancy, which may be referred to as unequal-probability-related redundancy. More explicitly, the so-called unequal-probability-related redundancy, which manifests itself in terms of the unequal probability of occurence of the $M$-ary source symbols is exploited as a priori information for computing the extrinsic LLR values. The extrinsic LLRs $L_{1, e}(u)$ of the speech parameters can be generated as detailed in [4].

The proposed scheme's performance was studied against its benchmarker, which does not employ the OCM. We will refer to the benchmarker as the DSTS-SP-RSC-AMRWB scheme. In the benchmark scheme advocated, the natural residual redundancy inherent in the AMR-WB encoded parameters was exploited, as detailed in [10].

In this paper, we investigate the effect of employing different combinations of RSC and OCM rates on the DSTS-SP-RSC-AMRWBOCM scheme of Figure 1. More explicitly, we investigate the effect of appropriately apportioning the redundancy among the channel encoder and OCM scheme.

Table 1 summarises the DSTS-SP-RSC-AMRWB-OCM schemes employing different combinations of RSC and OCM rates, while fixing the overall code rate $R_{\text {system }}$. More explicitly, we investigate the DSTS-SP-RSC-AMRWB-OCM schemes having two different overall coding rates, namely $R_{\text {system }}=1 / 2$ and $R_{\text {system }}=2 / 3$, but employing different combinations of RSC and OCM rates, as detailed in Table 1.

\begin{tabular}{|c|c|c|c|}
\hline $\begin{array}{c}\text { OCM-Aided AMR-WB } \\
\text { MIMO Transceiver }\end{array}$ & RSC Rate & OCM Rate & $\begin{array}{c}E_{b} / N_{0} \\
\text { Gain }(\mathrm{dB})\end{array}$ \\
\hline \hline \multicolumn{4}{|c|}{ System with $R_{\text {system }} \mathbf{= 1 / 2}$} \\
\hline \hline URC-Scheme 1 & 1 & $1 / 2$ & 0.3 \\
RSC-Scheme 1 & $2 / 3$ & $3 / 4$ & 1.0 \\
\hline \hline \multicolumn{4}{|c|}{ System with $R_{\text {system }} \mathbf{= 2 / 3}$} \\
\hline \hline URC-Scheme 2 & 1 & $2 / 3$ & 1.0 \\
RSC-Scheme 2 & $3 / 4$ & $8 / 9$ & 2.0 \\
\hline
\end{tabular}

Table 1: The DSTS-SP-RSC-AMRWB-OCM scheme of Figure 1 having different combinations of RSC and OCM rates, when $R_{\text {system }}=1 / 2$ and $R_{\text {system }}=2 / 3$.

\section{EXIT CHART ANALYSIS}

EXIT charts have been widely used in the design of iterative schemes, which facilitate the prediction of the associated decoding convergence behaviour, based on the exchange of mutual information amongst the constituent receiver components.

Observe in Figure 1 that the RSC exchanges appropriately interleaved information with both the SP and the AMR-WB decoders. More explicitly, the RSC decoder receives the a priori input $I_{2, A}(c)$ from the SP demapper, as well as the a priori input $I_{2, A}(\tilde{u})$ from the SBSD decoder. The variable $I_{2, A}(c)$ denotes the mutual information (MI) [11] between the a priori LLR values $L_{2, A}(c)$ and the corresponding coded bits $c$ originating from the extrinsic output of the SP demapper. On the other hand, the variable $I_{2, A}(\tilde{u})$ denotes the MI between the a priori LLR values $L_{2, A}(\tilde{u})$ and the data bits $\tilde{u}$, which was generated from the extrinsic output of the SBSD decoder.

Consequently, the extrinsic outputs $I_{2, E}(c)$ and $I_{2, E}(\tilde{u})$ are generated by the RSC decoder, where the corresponding EXIT functions are $T_{c}\left[I_{2, A}(\tilde{u}), I_{2, A}(c)\right]$ and

$T_{\tilde{u}}\left[I_{2, A}(\tilde{u}), I_{2, A}(c)\right]$, respectively. However, the EXIT characteristic of the SP demapper is dependent on the a priori input, $L_{3, A}(\tilde{c})$ and the $E_{b} / N_{0}$ value, while that of the SBSD decoder depends on only a single a priori input, $L_{1, A}(u)$. Therefore, the corresponding EXIT functions of the SP demapper and the SBSD decoder may be described by $T_{\tilde{c}}\left[I_{3, A}(\tilde{c}), E_{b} / N_{0}\right]$ and $T_{u}\left[I_{1, A}(u)\right]$, respectively.

The EXIT charts of the advocated systems of Table 1, namely the URC-Scheme 1, the RSC-Scheme 1, the URC-Scheme 2 and the RSC-Scheme 2, are shown in Figures 2, 3, 4 and 5, respectively. The joint EXIT function characterizes the best possible attainable performance, when exchanging information between the SP demapper and the RSC decoder of Figure 1 for different fixed values of $I_{2, A}(c)$, which is denoted by the line marked with squares. The line indicated by the triangles represents the EXIT curve of the AMR-WB decoder assisted by the OCM scheme, referred to as the AMRWB-OCM arrangement. We will refer to the joint EXIT function of the inner and the intermediate SISO modules of the DSTS-SP-RSC-AMRWBOCM scheme as the EXIT curve of the DSTS-SP-RSC block. Also shown in Figures 2, 3, 4 and 5 are the EXIT charts of the corresponding DSTS-SP-RSC-AMRWB benchmark scheme indicated by the dotted line.

As seen from Figures 2, 3, 4 and 5, the EXIT curve of the AMRWB decoder denoted by the dotted line marked with triangles, cannot reach the point of perfect convergence at $(1,1)$ and intersects with the EXIT curve of the DSTS-SP-RSC block, which implies that residual errors persist, regardless of both the number of iterations used and the size of the interleaver. On the other hand observe in Figures 2, 3, 4 
and 5 that by exploiting the intentional redundancy imposed by the OCM on the AMR-WB encoded bitstream allowed the AMRWBOCM scheme's EXIT curve to reach the point of perfect convergence at $(1,1)$. Therefore, it is predicted that the proposed scheme outperforms its benchmark arrangement also in terms of its Bit Error Ratio (BER).

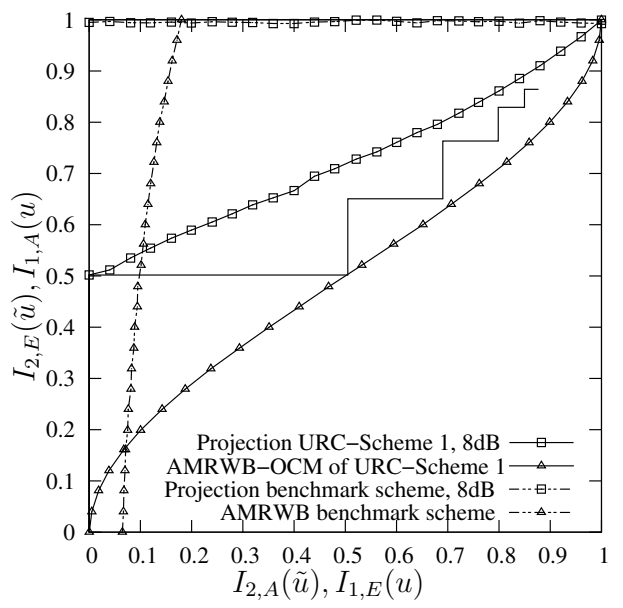

Figure 2: The EXIT chart of the URC-Scheme 1 of Table 1 at $E_{b} / N_{0}=8.0 \mathrm{~dB}$.

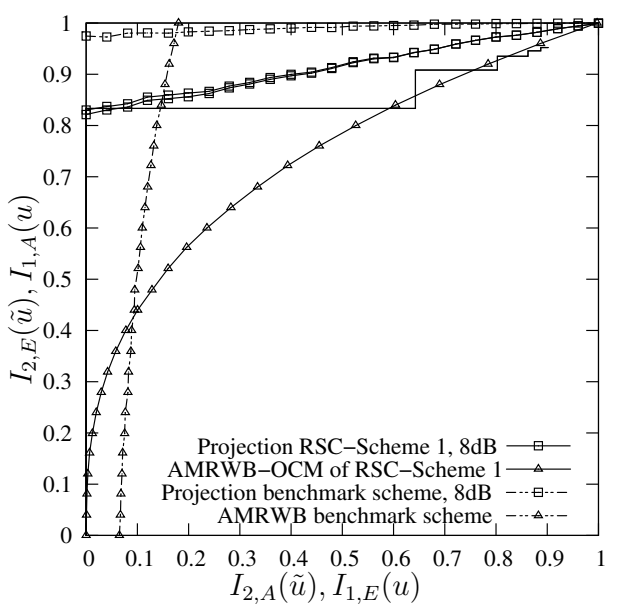

Figure 3: The EXIT chart of the RSC-Scheme 1 of Table 1 at $E_{b} / N_{0}=8.0 \mathrm{~dB}$

The actual decoding trajectories of the DSTS-SP-RSC-AMRWBOCM schemes of Table 1 recorded at $E_{b} / N_{0}=8 \mathrm{~dB}$ and $I_{\text {system }}=4$ iterations are also illustrated in the corresponding EXIT charts of Figures 2, 3, 4 and 5. However, the Monte-Carlo simulation-based iterative decoding trajectories do not closely follow the EXIT characteristics due to the short interleaver length employed.

We can observe in Figures 2 and 3 for the system having the overall code-rate of $R_{\text {system }}=1 / 2$ that both the URC-Scheme 1 and the RSC-Scheme 1 exhibit an open convergence tunnel at $E_{b} / N_{0}=8 \mathrm{~dB}$. However, as seen in Figure 2, the actual decoding trajectory of the URC-Scheme 1 recorded at $E_{b} / N_{0}=8 \mathrm{~dB}$ for $I_{\text {system }}=4$ iterations reaches the point $\left(I_{1, A}, I_{1, E}\right)=(0.86,0.88)$, while that of the RSCScheme 1 is capable of reaching a point closer to the $\left(I_{1_{A}}, I_{1, E}\right)=$ $(1,1)$, namely $\left(I_{1, A}, I_{1, E}\right)=(0.95,0.90)$. Thus, according to the EXIT chart predictions of Figures 2 and 3, as well as to the corre-

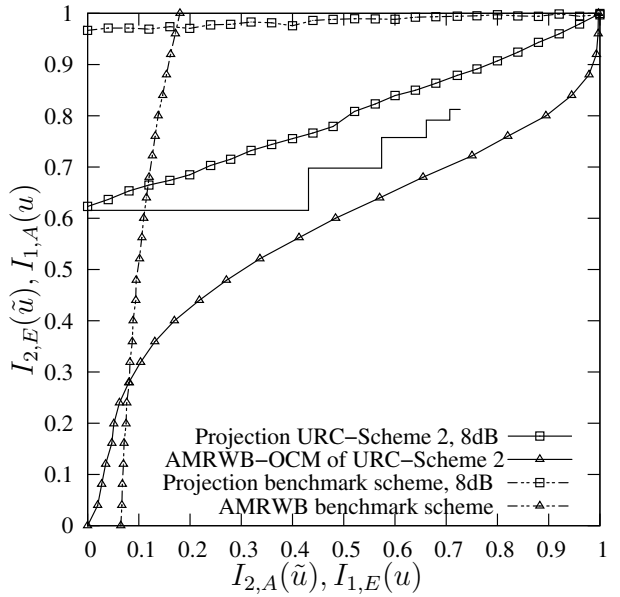

Figure 4: The EXIT chart of the URC-Scheme 2 of Table 1 at $E_{b} / N_{0}=8.0 \mathrm{~dB}$

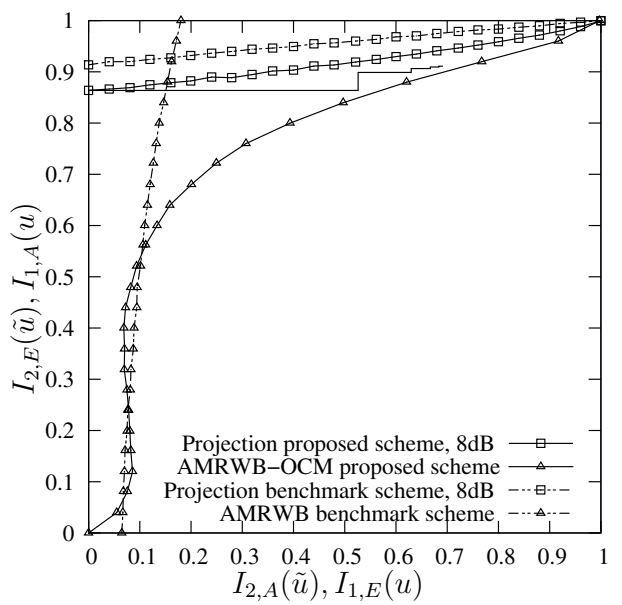

Figure 5: The EXIT chart of the RSC-Scheme 2 of Table 1 at $E_{b} / N_{0}=8.0 \mathrm{~dB}$.

sponding actual decoding trajectory, the RSC-Scheme 1 outperforms the URC-Scheme 1 after $I_{\text {system }}=4$ iterations. Similar observations may be made from Figures 4 and 5 for the system having the overall code-rate of $R_{\text {system }}=2 / 3$. Therefore, it was found to be beneficial to appropriately apportion the redundancy among the channel encoder and the OCM scheme, rather than assigning all the redundancy to the OCM scheme, despite the fact that they both exhibit an open convergence tunnel.

\section{BER AND SEGSNR PERFORMANCE RESULTS}

In this section, the attainable performance of the proposed scheme is characterised in terms of its BER and Segmental Signal to Noise Ratio (SegSNR) [12] evaluated at the speech decoder's output as a function of the channel Signal to Noise Ratio (SNR) per bit. Figures $6,7,8$ and 9 depict the BER versus $E_{b} / N_{0}$ performance of the URC-Scheme 1, of the RSC-Scheme 1, of the URC-Scheme 2 and of the RSC-Scheme 2 of Table 1, as well as that of their corresponding DSTS-SP-RSC-AMRWB benchmark schemes. It can be seen from Figures $6,7,8$ and 9 that at $\mathrm{BER}=1 \times 10^{-4}$, the URC-Scheme 1 , the RSC-Scheme 1, the URC-Scheme 2 and the RSC-Scheme 2 outperform their corresponding benchmark schemes in terms of $E_{b} / N_{0}$ by 


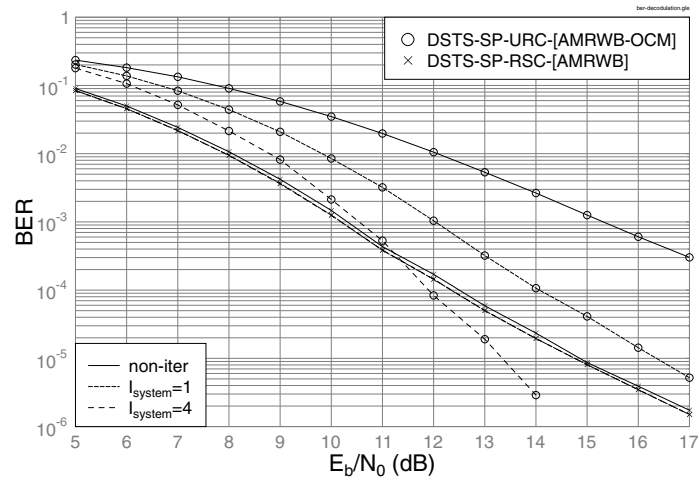

Figure 6: BER versus $E_{b} / N_{0}$ performance of the URC-Scheme 1 of Table 1.

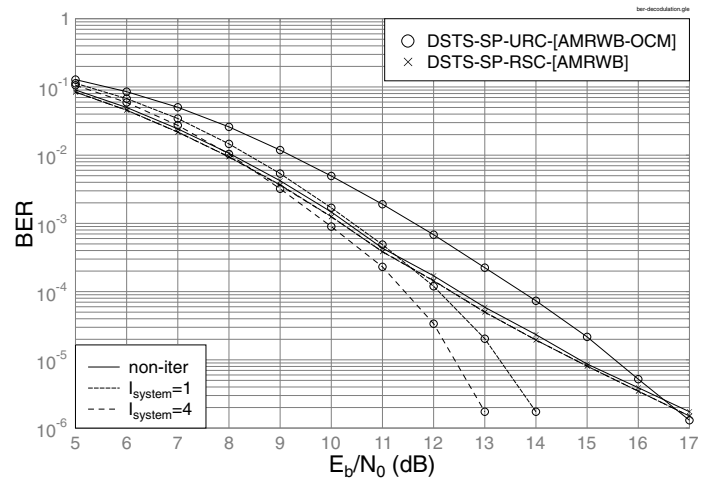

Figure 7: BER versus $E_{b} / N_{0}$ performance of the RSC-Scheme 1 of Table 1.

about $0.3 \mathrm{~dB}, 1.0 \mathrm{~dB}, 2.0 \mathrm{~dB}$ and $3.0 \mathrm{~dB}$, respectively, after $I_{\text {system }}=4$ iterations.

It can be seen from Figures 2 and 4 that a higher-rate OCM scheme requires a higher channel SNR for maintaining an open tunnel between the EXIT curve of the DSTS-SP-RSC and that of the AMRWB decoder assisted OCM, which is a prerequisite for the sake of avoiding persistent residual errors. This is reflected in the BER curve shown in Figures 6 and 8, respectively, where the URC-Scheme 1 and the URC-Scheme 2 required $E_{b} / N_{0}$ values of about $12 \mathrm{~dB}$ and $14.2 \mathrm{~dB}$, respectively, for achieving a BER of $1 \times 10^{-4}$.

The corresponding SegSNR performances are shown in Figures 10, 11,12 and 13 for $I_{\text {system }}=4$ iterations, where the URC-Scheme 1, the RSC-Scheme 1, the URC-Scheme 2 and the RSC-Scheme 2, outperformed their corresponding DSTS-SP-RSC-AMRWB benchmark schemes by approximately $0.3 \mathrm{~dB}, 1.0 \mathrm{~dB}, 1.0 \mathrm{~dB}$ and $2.0 \mathrm{~dB}$, respectively, when tolerating a SegSNR degradation of $1 \mathrm{~dB}$.

More explicitly, the intentionally imposed residual redundancy of the AMR-WB-encoded bitstream using the OCM scheme has improved the EXIT-characteristics of the soft-bit source decoder, which resulted in an enhanced attainable BER performance for the DSTSSP-RSC-AMRWB-OCM scheme. Although both the DSTS-SP-RSCAMRWB-OCM and the DSTS-SP-RSC-AMRWB schemes have the same overall coding rate of $R_{\text {system }}=R_{\text {benchmark }}$, the former assigns part of its channel encoder's redundancy to the OCM scheme and this is in addition to the source's residual redundancy inherited in the source-encoded bitstream. For example, in the RSC-Scheme 2 a 3/4-rate RSC code having a code memory of 3 was invoked, which resulted in the overall coding rate of $R_{\text {system }}=464 / 708 \approx 0.66$. On

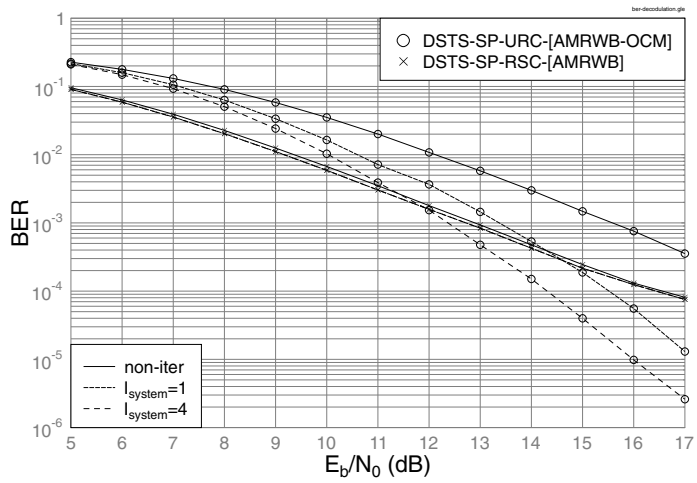

Figure 8: BER versus $E_{b} / N_{0}$ performance of the URC-Scheme 2 of Table 1.

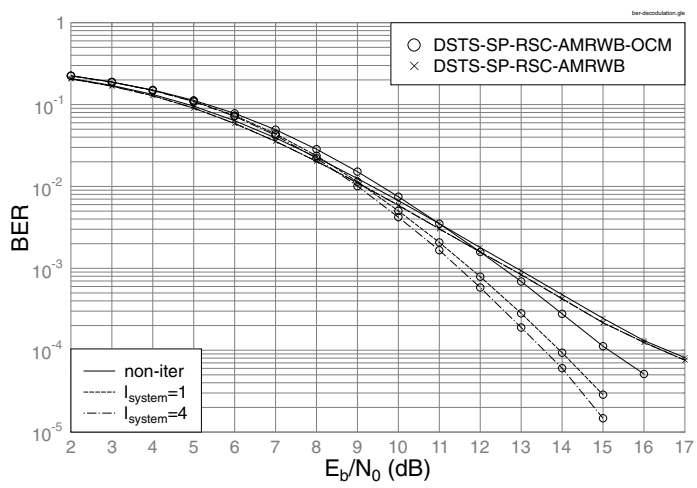

Figure 9: BER versus $E_{b} / N_{0}$ performance of the RSC-Scheme 2 of Table 1.

the other hand, the corresponding DSTS-SP-RSC-AMRWB benchmark scheme employed a 2/3-rate RSC code having a code memory of 4 but dispensing with the OCM scheme, resulted in the overall coding rate of $R_{\text {benchmark }}=464 / 708 \approx 0.66$. It was shown in Figures 5 of Section 3 that the DSTS-SP-RSC-AMRWB-OCM scheme benefits from "an early" convergence, when the total available redundancy was appropriately apportioned for the OCM and channel encoders. More explicitly, the employment of the OCM scheme created an open EXIT chart tunnel right through to the convergence point of $(1,1)$ even at a low SNR, as shown in Figure 5 of Section 3. The achievable performance was also studied against that of the benchmark scheme, where the redundancy was assigned entirely to the channel encoder.

In the case when the advocated schemes have a fixed overall code rate of $R_{\text {system }}=1 / 2$, it can be observed in Figure 3 that the specific scheme which apportions the redundancy among the channel encoder and OCM scheme results in a beneficial improvement. More explicitly, the URC-Scheme 1 assigned all the redundancy to the OCM scheme, while the RSC-Scheme 1 employed a 1/2-rate RSC code combined with a 3/4-rate OCM scheme. Similar observations may be made for the scheme having a fixed overall code rate of $R_{\text {system }}=2 / 3$.

Hence, our results demonstrate that a powerful joint source and channel coding schemes can be designed by employing iterative detection, as well as by appropriately apportioning the redundancy between the source and channel codecs.

\section{CONCLUSIONS}

The effect of employing different combinations of RSC and OCM rates on the DSTS-SP-RSC-AMRWB-OCM scheme was addressed in 


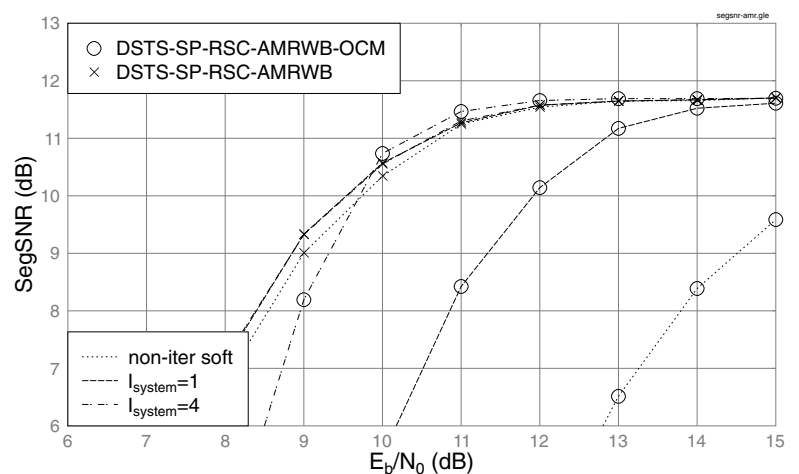

Figure 10: SegSNR versus $E_{b} / N_{0}$ performance of the URC-Scheme 1 of Table 1.

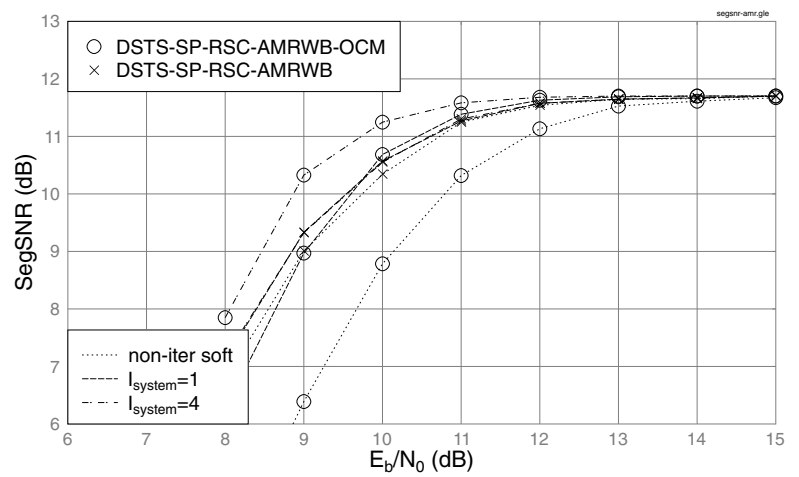

Figure 11: SegSNR versus $E_{b} / N_{0}$ performance of the RSC-Scheme 1 of Table 1.

this paper, while fixing the overall code-rate. More specifically, two systems having two different overall code rates, namely $R_{\text {system }}=1 / 2$ and $R_{\text {system }}=2 / 3$, but having different combinations of RSC and OCM rates were investigated, as summarised in Table 1 . It was found that the system that carefully apportioned the redundancy among the channel encoder and the OCM scheme provided a high system performance, when compared to the system that assigned all the redundancy to the OCM scheme, despite the fact that they both exhibited an open convergence tunnel. It was also demonstrated that the redundancy delibrately imposed on the AMR-WB-encoded bitstream using the OCM scheme provided a significant $E_{b} / N_{0}$ gain, when compared to its corresponding benchmark scheme dispensing with OCM, as summarised in Table 1.

\section{REFERENCES}

[1] J. Kliewer and R. Thobaben, "Iterative Joint Source-Channel Decoding of Variable-Length Codes Using Residual Source Redundancy," IEEE Transactions on Wireless Communications, vol. 4, pp. 919 - 929, May 2005.

[2] M. Adrat and P. Vary, "Iterative Source-Channel Decoding: Improved System Design Using EXIT Charts," EURASIP Journal on Applied Signal Processing, pp. 1727-1737, October 2005.

[3] T. Clevorn, M. Adrat and P. Vary, "Turbo Decodulation Using Highly Redundant Index Assignments and Multi-Dimensional Mappings," 4th Int. Symposium on Turbo Codes and Related Topics in connection with 6th Int. ITG-Conference on Source and Channel Coding, CD Rom, April 2006.

[4] M. Adrat, P. Vary, and J. Spittka, "Iterative Source-Channel Decoder Using Extrinsic Information from Softbit-Source Decoding," IEEE International Conference on Acoustics, Speech and Signal Processing, vol. 4, pp. 2653-2656, 7-11 May 2001.

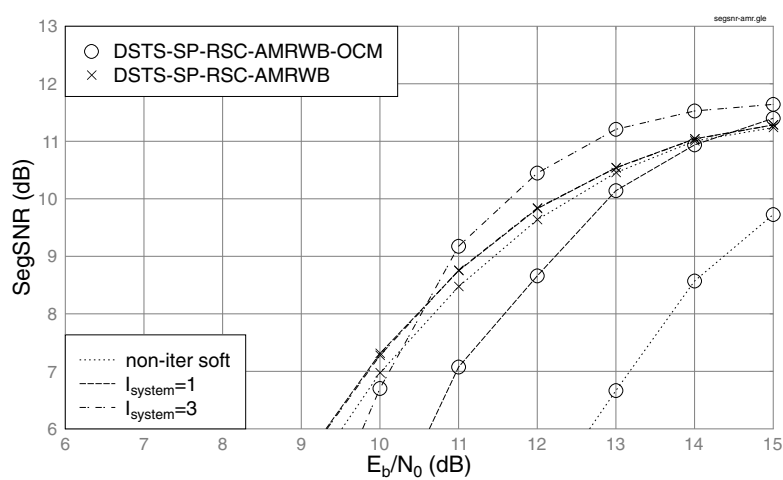

Figure 12: SegSNR versus $E_{b} / N_{0}$ performance of the URC-Scheme 2 of Table 1.

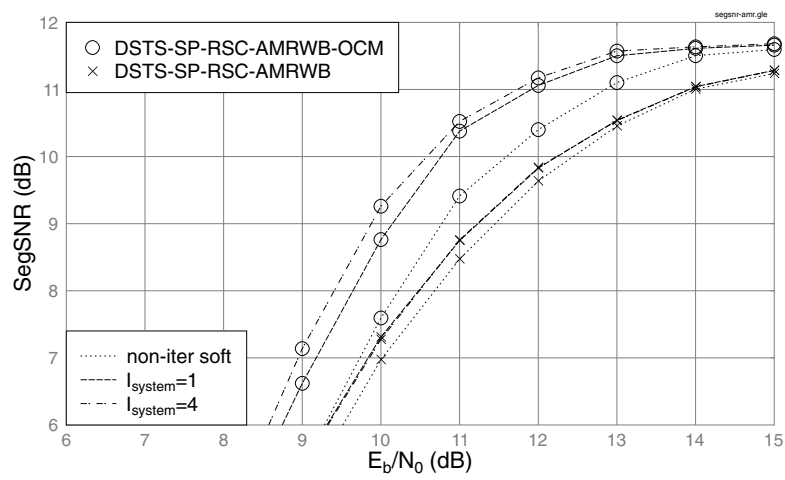

Figure 13: SegSNR versus $E_{b} / N_{0}$ performance of the RSC-Scheme 2 of Table 1.

[5] S. ten Brink, "Convergence Behaviour of Iteratively Decoded Parallel Concatenated Codes," IEEE Transactions on Communications, vol. 49, pp. 1727-1737, October 2001.

[6] N. S. Othman, M. El-Hajjar, A. Q. Pham, O. Alamri, S. X. Ng and L. Hanzo, "Over-Complete Source-Mapping Aided AMR-WB MIMO Transceiver Using Three-Stage Iterative Detection," in IEEE International Conference on Communications, pp. 751-755, 19-23 May 2008.

[7] B. Bessette, R. Salami, R. Lefebvre, M. Jelinek, J. Rotola-Pukkila, J. Vainio, H. Mikkola and K. Jarvinen, "The Adaptive Multirate Wideband Speech Codec (AMR-WB)," IEEE Transactions on Speech and Audio Processing, vol. 10, pp. 620-636, November 2002.

[8] A. Q. Pham, L. Hanzo and L. -L. Yang, "Joint Optimization of Iterative Source and Channel Decoding Using Over-Complete Source-Mapping," in IEEE 66th Vehicular Technology Conference, pp. 1072 - 1076, 30 Sept.-3 Oct. 2007.

[9] M. El-Hajjar, O. Alamri, S. X. Ng and L. Hanzo, "Turbo Detection of Precoded Sphere Packing Modulation Using Four Transmit Antennas for Differential Space-Time Spreading," IEEE Transactions on Wireless Communications, vol. 7, pp. 943-952, March 2008.

[10] N. S. Othman, M. El-Hajjar, O. Alamri and L. Hanzo, "Soft-Bit Assisted Iterative AMR-WB Source-Decoding and Turbo-Detection of ChannelCoded Differential Space-Time Spreading Using Sphere Packing Modulation," IEEE 65th Vehicular Technology Conference, pp. 2010-2014, 22-25 April 2007.

[11] C. E. Shannon, "A Mathematical Theory of Communication," The Bell System Technical Journal, vol. 27, pp. 379-423,623-656, July, October 1948.

[12] L. Hanzo, F. C. A. Somerville and J. P. Woodard, Voice and Audio Compression for Wireless Communications, 2nd Edition. Chichester, UK: John Wiley-Sons Inc., 2007. 